\title{
Plasma concentrations of zonulin are elevated in obese men with fatty liver disease
}

\author{
A-Sol Kim ${ }^{1,2}$ \\ Hae-Jin Ko ${ }^{1,3}$ \\ 'Department of Family Medicine, \\ School of Medicine, Kyungpook \\ National University, Daegu, South \\ Korea; ${ }^{2}$ Department of Family \\ Medicine, Kyungpook National \\ University Chilgok Hospital, Daegu, \\ South Korea; ${ }^{3}$ Department of Family \\ Medicine, Kyungpook National \\ University Hospital, Daegu, South \\ Korea
}

This article was published in the following Dove Press journal: Diabetes, Metabolic Syndrome and Obesity:Targets and Therapy

Purpose: Zonulin is considered as a biomarker of increased intestinal permeability. The relationship between intestinal permeability and obesity is known, and many studies have investigated the relationship between intestinal permeability and liver disease. Thus, we aimed to investigate the potential association between plasma zonulin concentrations and fatty liver in obese men.

Patients and methods: A total of 140 obese men without inflammatory bowel diseases, autoimmune diseases, and severe liver diseases were included. The subjects were divided into three groups: normal, mild fatty liver, and moderate-to-severe fatty liver, according to abdominal ultrasonography findings. We subdivided the subjects into two subgroups based on the amount of alcohol consumption (appropriate drinking and hazardous drinking), and subgroup analyses were performed.

Results: The mean plasma zonulin concentrations $(\mathrm{ng} / \mathrm{mL})$ in the normal, mild fatty liver, and moderate-to-severe fatty liver groups were $0.618,2.143$, and 5.815 , respectively $(P<0.001)$. A multivariate multinomial logistic regression analysis revealed an odds ratio (OR) of 1.77 $(P=0.015)$ in the moderate-to-severe fatty liver group. The median plasma zonulin concentrations $(\mathrm{ng} / \mathrm{mL})$ in the appropriate drinking subgroup of the fatty liver groups were $0.002,0.500$, and 6.550, respectively ( $P$-trend $<0.001)$, and in the hazardous drinking subgroup were $0.002,0.590$, and 5.800, respectively $(P$-trend $=0.001)$. The ORs for moderate-to-severe fatty liver were 1.91 $(P=0.039)$ in the appropriate drinking group and $1.56(P=0.045)$ in the hazardous drinking group. Conclusion: Plasma zonulin concentrations were elevated among obese men. A significant association was found between zonulin concentrations and severity of fatty liver.

Keywords: zonulin, intestinal permeability, fatty liver, obesity

\section{Introduction}

Intestine is an organ that digests and absorbs nutrients and also acts as a barrier against harmful foreign substances. Intestinal epithelial cells form a tight junction with the surrounding cells, and a fixed gap is maintained that functions as a primary barrier against bacteria, viruses, toxins, and allergens that enter the body through the digestive system. ${ }^{1}$

When the intestine becomes irritated or damaged, the tight junctions weaken and

Correspondence: Hae-jin Ko

Department of Family Medicine, Kyungpook National University Hospital, I 30 Dongdeok-ro, Jung-gu, 41944 Daegu, South Korea

Tel +82532006578

Fax +82532005480

Email liveforme@knu.ac.kr intestinal permeability increases, which permits macromolecules to enter and exit through the gap. ${ }^{2}$ With the increase in intestinal permeability, the intestine fails to act as a primary barrier, which can lead to various clinical symptoms. Intestinal permeability significantly increases in intestinal disease states such as celiac disease. ${ }^{3}$ Liver diseases and metabolic diseases such as obesity and insulin resistance can also increase intestinal permeability. ${ }^{4,5}$ Thus, intestinal permeability has received considerable attention from researchers. 
Fatty liver disease and obesity are closely associated. Fatty liver disease refers to the excessive accumulation of fat in the liver due to alcoholic or nonalcoholic causes. It is an important risk factor for cirrhosis and liver cancer. It is also closely associated with major metabolic disorders, such as insulin resistance, diabetes, hypertension, and dyslipidemia. ${ }^{6}$ The prevalence of fatty liver increases with age and is higher in men than in women. ${ }^{7}$ Alcohol consumption and obesity are major risk factors for fatty liver disease. The risk for developing metabolic syndrome increases when an obese person develops fatty liver disease, and consequently, the risk for cardiovascular diseases and death can be enhanced. ${ }^{8}$ Greater intestinal permeability leads to increased levels of plasma lipopolysaccharides (LPS) that can cause low-grade inflammation and heighten the risk of insulin resistance and metabolic disorders. ${ }^{9}$ Research on the intestinal permeability of obese patients with fatty liver disease may have important implications in health care, but such research is lacking. Several previous studies have reported increased intestinal permeability in patients with serious liver diseases, such as cirrhosis, ${ }^{10}$ and have noted the changes in the gut microbiota and increased intestinal permeability in obese diabetic patients. ${ }^{11}$ However, all the studies were conducted in Western countries and no research had specifically considered people who were obese.

Intestinal permeability can be assessed by several methods. Plasma zonulin concentrations are frequently used to assess intestinal permeability as they can be easily measured using blood tests. ${ }^{12}$ Zonulin is a protein that reversibly controls intestinal permeability by controlling the binding between the epithelial cells of the intestinal mucosa. ${ }^{13}$ It is involved in the innate intestinal immunity, ${ }^{14}$ and its expression is upregulated in patients with celiac disease and type 1 diabetes. ${ }^{12,15}$ Plasma zonulin concentrations are strongly correlated with the lactulose-mannitol ratio, which was earlier used to measure intestinal permeability in clinical settings. ${ }^{5}$

This study was designed to analyze only men with a high prevalence of fatty liver and a high risk of hazardous drinking. We analyzed the correlation between plasma zonulin concentrations and fatty liver disease in relatively healthy obese men identified during medical checkups for the prevention and management of metabolic diseases.

\section{Patients and methods}

\section{Study subjects}

Male patients who visited a university hospital in Daegu, South Korea, between October 2015 and September 2016 for a medical check-up and who met the following inclusion criteria were enrolled: older than 30 years but younger than 60 years, body mass index (BMI) of $\geq 25 \mathrm{~kg} / \mathrm{m}^{2}$, and having abdominal obesity (waist circumference of $\geq 90 \mathrm{~cm}$ ). Patients meeting the following exclusion criteria were excluded from the study: estimated glomerular filtration rate (eGFR) of $<60 \mathrm{~mL} / \mathrm{min} / 1.73 \mathrm{~m}^{2}$, previous history of autoimmune diseases such as rheumatoid arthritis and systemic lupus erythematosus, currently receiving chemotherapy or having received chemotherapy in the last year, consumption of alcoholic beverages within 12 hours prior to evaluation, and a history of severe liver diseases such as cirrhosis, celiac disease, inflammatory bowel disease, and uncontrollable irritable bowel disease. The total number of participants who agreed to participate in the study and satisfied the inclusion and exclusion criteria was 140 initially.

This study was reviewed and approved by the institutional review board of Kyungpook National University Hospital. Written informed consent was obtained from all individual participants included in the study.

\section{Demographics}

Age, sex, medical history, drinking habits, smoking habits, and level of physical activity were obtained through selfreported questionnaires completed by patients during their visits to the health center. Participants were classified as current smokers, former smokers, and nonsmokers based on their responses. Regarding drinking habits, the amount of alcohol consumption was measured via questionnaires. In accordance with the alcohol consumption classification developed by the Centers for Disease Control and Prevention, the participants were classified as nondrinkers if they had less than two standard drinks per week, social drinkers if they had more than three and $<14$ standard drinks per week, heavy drinkers if they had more than 15 standard drinks per week, and binge drinkers if they had more than five standard drinks in one setting. The participants were classified as active if they performed $>150$ minutes of moderately intense exercise every week.

\section{Body and obesity measurement}

The participants' heights and weights were measured using an automatic height measurement instrument, and the BMI $\left(\mathrm{kg} / \mathrm{m}^{2}\right)$ was calculated from these values. Waist circumference was horizontally measured from the bottom of the lowest rib to the middle of the top part of the iliac crest with a tape, without applying pressure on the soft tissue. 


\section{Physical examination and laboratory test}

Systolic and diastolic blood pressures were measured using an automatic sphygmomanometer after allowing the patient to sit and relax for at least 10 minutes. Blood sample was collected after the patient had fasted for at least 8 hours. Complete blood count, liver function (aspartate aminotransferase [AST], alanine aminotransferase [ALT], gamma-glutamyltransferase [GGT], total bilirubin [TB]), fasting blood glucose, HbAlc, lipid profile (total cholesterol, low-density lipoprotein cholesterol [LDL-C], high-density lipoprotein cholesterol [HDL-C], triglyceride), and creatinine were measured. eGFR was calculated using the Modification of Diet in Renal Disease (MDRD) Study equation. ${ }^{16}$

\section{Plasma zonulin concentrations}

Plasma zonulin concentrations were measured using an enzyme-linked immunosorbent assay (Immundiagnostik AG, Bensheim, Germany). The sensitivity of the assay was $<0.01 \mathrm{ng} / \mathrm{mL}$.

\section{Assessment of fatty liver disease}

Abdominal ultrasonography was performed by a radiologist after an 8-hour fast. The severity of fatty liver disease was classified into four stages based on echogenicity, the echoic tissue amplitude of the liver and kidneys, the degree of echo penetration, and the degree to which the vascular structures of the liver were defined. Patients were defined by the following classifications: normal, if they showed normal echogenicity (Grade 0); mild fatty liver, if the echogenicity of the hepatic parenchyma was slightly increased and the vein walls and diaphragm were clearly observed (Grade 1); moderate fatty liver, if echogenicity of hepatic parenchyma was moderately increased and the vein walls and diaphragm were only somewhat observed (Grade 2); and severe fatty liver, if the echogenicity of hepatic parenchyma was significantly increased, the vein walls and diaphragm could not be observed, and the right posterior segment was only minimally visible due to clear acoustic attenuation (Grade 3). ${ }^{17}$

\section{Statistical analyses}

Participants were divided into three groups (normal, mild fatty liver, and moderate-to-severe fatty liver) according to the severity of fatty liver. One-way analysis of variance (ANOVA) and Pearson's chi-square test were used to compare the demographic characteristics, laboratory test results, and plasma zonulin concentrations among the three groups. Multinomial logistic regression was performed to analyze the associations between plasma zonulin concentrations and fatty liver disease.

The nondrinkers and the social drinkers were assigned to the appropriate drinking subgroup, and the heavy and binge drinkers were assigned to the hazardous drinking subgroup, since drinking habits are an important factor of fatty liver disease. The Kruskal-Wallis test and multinomial logistic regression were performed for each subgroup to analyze the associations between plasma zonulin concentrations and fatty liver disease. All statistical analyses were performed using SPSS version 23 (IBM Corporation, Armonk, NY, USA). A $P$-value of $<0.05$ was considered as statistically significant.

\section{Results}

\section{Demographics}

Of the 140 total participants, 17 had Grade 0 classification and were assigned to the normal group, 89 had Grade 1 classification and were assigned to the mild fatty liver group, and the remaining 34 had Grade 2 or 3 classification and were assigned to the moderate-to-severe fatty liver group. The mean weight, BMI, and waist circumference measurements increased as the severity of fatty liver increased, but this was not statistically significant. No significant difference was found in the fasting blood glucose levels or $\mathrm{HbAlc}$ concentrations between the groups. No significant difference was found in the levels of the liver function markers (AST, ALT, GGT, and TB).

Total cholesterol, triglyceride, and LDL-C levels were not significantly different between the groups. However, the mean HDL-C levels were 49.0 $\pm 9.1,47.0 \pm 8.9$, and $42.9 \pm 9.2 \mathrm{mg} / \mathrm{dL}$ in the normal, mild fatty liver, and moderate-to-severe fatty liver groups, respectively, and significantly differed between the groups $(P=0.035)$.

No significant difference was found in the level of alcohol consumption, smoking, and physical activity between the groups. No significant difference was found in the medical histories of hypertension, diabetes, and dyslipidemia between the groups (Table 1).

\section{Plasma zonulin concentrations}

The mean plasma zonulin concentrations were $0.618 \pm 1.15$, $2.143 \pm 4.27$, and $5.815 \pm 3.11 \mathrm{ng} / \mathrm{mL}$ in the normal, mild fatty liver, and moderate-to-severe fatty liver groups, respectively, and these values significantly differed between the groups. Zonulin concentrations tended to increase as the severity of fatty liver disease increased $(P$-trend $<0.001$; Table 1$)$. 
Table I Clinical characteristics of the subjects

\begin{tabular}{|c|c|c|c|c|}
\hline Characteristic & $\begin{array}{l}\text { Normal } \\
(n=17)\end{array}$ & $\begin{array}{l}\text { Mild fatty liver } \\
(n=89)\end{array}$ & $\begin{array}{l}\text { Moderate-to-severe } \\
\text { fatty liver }(n=34)\end{array}$ & $P$-value \\
\hline Age, years & $44.5 \pm 9.5$ & $44.8 \pm 8.9$ & $44.7 \pm 9.2$ & 0.986 \\
\hline Systolic BP, mmHg & $132.9 \pm 1 \mid .3$ & $131.0 \pm 16.6$ & $132.5 \pm 12.1$ & 0.830 \\
\hline Diastolic BP, mmHg & $82.3 \pm 8.6$ & $81.9 \pm 11.4$ & $84.8 \pm I I .4$ & 0.433 \\
\hline Height, cm & $173.8 \pm 8.4$ & $174.3 \pm 6.5$ & $174.9 \pm 4.8$ & 0.838 \\
\hline Weight, kg & $84.1 \pm 10.7$ & $87.3 \pm 10.6$ & $89.8 \pm 9.4$ & 0.168 \\
\hline $\mathrm{BMI}, \mathrm{kg} / \mathrm{m}^{2}$ & $27.8 \pm 2.5$ & $28.8 \pm 2.8$ & $29.3 \pm 2.6$ & 0.161 \\
\hline WC, $\mathrm{cm}$ & $96.3 \pm 4.8$ & $97.2 \pm 6.5$ & $97.5 \pm 6.0$ & 0.806 \\
\hline Fasting glucose, $\mathrm{mg} / \mathrm{dL}$ & $98.6 \pm 13.9$ & $101.4 \pm 16.4$ & $100.0 \pm 23.9$ & 0.818 \\
\hline $\mathrm{HbAlc}, \mathrm{g} / \mathrm{dL}$ & $5.6 \pm 0.5$ & $5.8 \pm 0.8$ & $7.1 \pm 7.7$ & 0.205 \\
\hline AST, U/L & $25.5 \pm 11.4$ & $26.1 \pm 14.5$ & $27.9 \pm 17.6$ & 0.802 \\
\hline $\mathrm{ALT}, \mathrm{U} / \mathrm{L}$ & $30.0 \pm 15.2$ & $37.2 \pm 20.6$ & $38.7 \pm 27.9$ & 0.391 \\
\hline GGT, U/L & $65.5 \pm 55.1$ & $62.0 \pm 84.4$ & $58.9 \pm 48.0$ & 0.954 \\
\hline $\mathrm{TB}, \mathrm{mg} / \mathrm{dL}$ & $0.78 \pm 0.24$ & $0.87 \pm 0.37$ & $0.86 \pm 0.4 I$ & 0.695 \\
\hline Total cholesterol, mg/dL & $180.1 \pm 43.8$ & $192.6 \pm 35.8$ & $186.4 \pm 35.8$ & 0.373 \\
\hline LDL-C, mg/dL & $115.0 \pm 34.7$ & $135.5 \pm 31.7$ & $134.6 \pm 32.2$ & 0.055 \\
\hline $\mathrm{HDL}-\mathrm{C}, \mathrm{mg} / \mathrm{dL}$ & $49.0 \pm 9.1$ & $47.0 \pm 8.9$ & $42.9 \pm 9.2$ & 0.035 \\
\hline Triglyceride, mg/dL & $174.2 \pm 86.3$ & $173.3 \pm 73.3$ & $153.4 \pm 39.2$ & 0.336 \\
\hline Serum creatinine, $\mathrm{mg} / \mathrm{dL}$ & $0.90 \pm 0.18$ & $0.89 \pm 0.13$ & $0.91 \pm 0.13$ & 0.704 \\
\hline eGFR, $\mathrm{mL} / \mathrm{min} / 1.73 \mathrm{~m}^{2}$ & $95.6 \pm 23.1$ & $95.3 \pm 14.5$ & $91.1 \pm 25.0$ & 0.513 \\
\hline Zonulin, ng/mL $\mathrm{L}^{\mathrm{a}}$ & $0.618 \pm 1.15$ & $2.143 \pm 4.27$ & $5.815 \pm 3.11$ & $<0.001$ \\
\hline Smoking status & & & & 0.466 \\
\hline Nonsmoker & $2(11.8)$ & $16(18.0)$ & $10(29.4)$ & \\
\hline Former smoker & $9(52.9)$ & $38(42.7)$ & II (32.4) & \\
\hline Current smoker & $6(35.3)$ & $35(32.4)$ & $13(38.2)$ & \\
\hline Alcohol consumption & & & & 0.476 \\
\hline None & $\mathrm{I}(5.9)$ & $9(10.1)$ & $7(20.6)$ & \\
\hline Social & $9(52.9)$ & $35(39.3)$ & $7(20.6)$ & \\
\hline Heavy & $6(35.3)$ & $32(36.0)$ & II (32.4) & \\
\hline Binge & I (5.9) & $13(14.6)$ & $9(26.5)$ & \\
\hline \multicolumn{5}{|l|}{ Physical activity } \\
\hline Inactive & $8(47.1)$ & $48(53.9)$ & $15(44.1)$ & \\
\hline Active & $9(52.9)$ & $4 I(46.1)$ & $19(55.9)$ & 0.591 \\
\hline \multicolumn{5}{|l|}{ Medical history } \\
\hline Hypertension & $6(35.3)$ & $21(23.6)$ & $8(23.5)$ & 0.579 \\
\hline Diabetes & $2(11.8)$ & $7(7.9)$ & $2(5.9)$ & 0.763 \\
\hline Dyslipidemia & $2(11.8)$ & $5(5.6)$ & $2(5.9)$ & 0.632 \\
\hline
\end{tabular}

Notes: All values are presented as mean \pm SD or number (\%). P-values were calculated using the chi-square test for discrete outcomes and one-way ANOVA for continuous outcomes. ${ }^{\text {TThe }}$ was a significant trend toward increased mean values of concentrations $(P$-trend $<0.00 \mathrm{I})$.

Abbreviations: BP, blood pressure; BMI, body mass index; WC, waist circumference; AST, aspartate aminotransferase; ALT, alanine aminotransferase; GGT, gammaglutamyltransferase; TB, total bilirubin; LDL-C, low-density lipoprotein cholesterol; HDL-C, high-density lipoprotein cholesterol; eGFR, estimated glomerular filtration rate; ANOVA, analysis of variance.

\section{Relative risk of fatty liver disease in relation to plasma zonulin concentrations}

A multinomial logistic regression analysis was performed to determine the associations between plasma zonulin concentrations and the severity of fatty liver disease. Model 1 produced a relative risk without controlling for any variables. Model 2 considered the univariate analysis results and produced a relative risk adjusted for BMI, $\mathrm{HbAlc}, \mathrm{LDL}-\mathrm{C}$, and HDL-C. Model 3 was adjusted for additional factors (age, drinking habits, smoking status, use of diabetes medications, and use of dyslipidemia medications) that have been reported to affect the incidence of fatty liver, in addition to the factors controlled in Model 2.

For mild fatty liver, Models 1, 2, and 3 did not produce statistically significant results. However, the relative risk of moderate-to-severe fatty liver significantly increased with the plasma zonulin concentrations in Model 1 (odds ratio $[\mathrm{OR}]=1.90,95 \% \mathrm{CI}=1.23-2.92)$, Model $2(\mathrm{OR}=1.83,95 \%$ 
$\mathrm{CI}=1.18-2.84)$, and Model $3(\mathrm{OR}=1.77,95 \% \mathrm{CI}=1.13-2.76$; Table 2).

\section{Differences in plasma zonulin concentrations according to the severity of fatty liver disease in alcohol consumption subgroups}

The appropriate drinking subgroup, comprising nondrinkers and social drinkers, consisted of 68 participants. Further classification of these participants according to the severity of fatty liver led to placement of 10 participants in the normal group, 44 participants in the mild fatty liver group, and 14 participants in the moderate-to-severe fatty liver group. The median plasma zonulin concentrations were $0.002,0.500$, and $6.550 \mathrm{ng} / \mathrm{mL}$ in each group. The plasma zonulin concentrations increased as the severity of fatty liver disease increased ( $P$-trend $=0.002)$.

The hazardous drinking subgroup, comprising heavy and binge drinkers, consisted of 72 participants. Further classification according to the severity of fatty liver disease resulted in 7, 45, and 20 participants in the normal, mild fatty liver, and moderate-to-severe groups, respectively. The median zonulin concentration increased as the severity of fatty liver disease increased, as was the case in the appropriate

Table 2 Effect of plasma zonulin concentrations on the risk of fatty liver disease

\begin{tabular}{|c|c|c|c|c|}
\hline & \multicolumn{2}{|l|}{ Mild fatty liver } & \multicolumn{2}{|c|}{$\begin{array}{l}\text { Moderate-to-severe } \\
\text { fatty liver }\end{array}$} \\
\hline & OR (95\% CI) & $P$-value & OR (95\% Cl) & $P$-value \\
\hline Model Ia & I.5I (0.99-2.30) & 0.059 & $1.90(1.23-2.92)$ & 0.004 \\
\hline Model $2^{b}$ & $1.44(0.94-2.22)$ & 0.095 & $1.83(1.18-2.84)$ & 0.007 \\
\hline Model $3^{c}$ & $1.40(0.9 \mid-2.16)$ & 0.122 & $1.77(1.13-2.76)$ & 0.015 \\
\hline
\end{tabular}

Notes: aUnadjusted model. 'BAdjusted for HbAlc, low-density lipoprotein cholesterol, high-density lipoprotein cholesterol, and BMI. 'Adjusted for the covariates in Model 2 as well as age, smoking, alcohol consumption, physical activity, diabetes, and dyslipidemia.

Abbreviations: OR, odds ratio; BMI, body mass index. drinking subgroup (median concentrations of 0.002, 0.590, and $5.800 \mathrm{ng} / \mathrm{mL}$, respectively; $P$-trend $=0.001$; Table 3 ).

\section{Zonulin concentrations as a risk factor of fatty liver disease in alcohol consumption subgroups}

A multivariate multinomial logistic regression analysis adjusted for BMI, HbA1c, LDL-C, HDL-C, age, alcohol consumption, smoking status, exercise, and use of diabetes and dyslipidemia medications was performed for each subgroup. In the case of the appropriate drinking subgroup, the relative risk of mild fatty liver and plasma zonulin concentrations were not significantly associated (adjusted OR $=1.63,95 \%$ $\mathrm{CI}=0.75-3.55)$. However, the relative risk of moderate-tosevere fatty liver increased as plasma zonulin concentrations increased (adjusted OR=1.91, 95\% CI=1.01-3.95; Figure 1).

Similarly, in the hazardous drinking subgroup, although the relative risk of mild fatty liver was not significantly associated with plasma zonulin concentrations (adjusted $\mathrm{OR}=1.17$, 95\% CI=0.69-1.96), the relative risk of moderate-to-severe fatty liver increased as plasma zonulin concentrations increased (adjusted $\mathrm{OR}=1.56,95 \% \mathrm{CI}=1.02-2.67$; Figure 2).

\section{Discussion}

The association between obesity and intestinal permeability has been clarified in numerous studies. Lam et al ${ }^{18}$ reported that high-fat diets increase the concentrations of Gramnegative bacteria containing LPS on the intestinal walls. This increase in concentration decreases the expression of zonula occludens-1 and occludin, which maintain the tight junction between intestinal epithelial cells, thus leading to increased intestinal permeability. Muccioli et a ${ }^{19}$ reported that the consumption of a high-fat diet and the subsequent changes in the gut microbiota induce changes in the endocannabinoid system, which also lead to increased intestinal permeability. MorenoNavarrete et $\mathrm{al}^{9}$ reported that plasma zonulin concentrations are elevated in obese persons. They suggested that increased

Table 3 Median concentration of plasma zonulin according to fatty liver severity in the subgroups

\begin{tabular}{|c|c|c|c|c|}
\hline \multicolumn{5}{|c|}{ Appropriate drinking subgroup } \\
\hline Normal $(n=10)$ & Mild fatty liver $(n=44)$ & Moderate-to-severe fatty liver $(n=\mid 4)$ & $P$-value & P-trend \\
\hline $0.002(0.00 \mathrm{I}-0.687)$ & $0.500(0.002-2.250)$ & $6.550(3.150-8.050)$ & $<0.001$ & 0.002 \\
\hline \multicolumn{5}{|c|}{ Hazardous drinking subgroup } \\
\hline Normal $(n=7)$ & Mild fatty liver $(n=45)$ & Moderate-to-severe fatty liver $(n=20)$ & $P$-value & P-trend \\
\hline $0.002(0.001-2.000)$ & $0.590(0.003-2.750)$ & $5.800(3.450-7.775)$ & $<0.001$ & 0.001 \\
\hline
\end{tabular}

Notes: All values are presented as median (interquartile range). P-values were calculated using the Kruskal-Wallis test. 


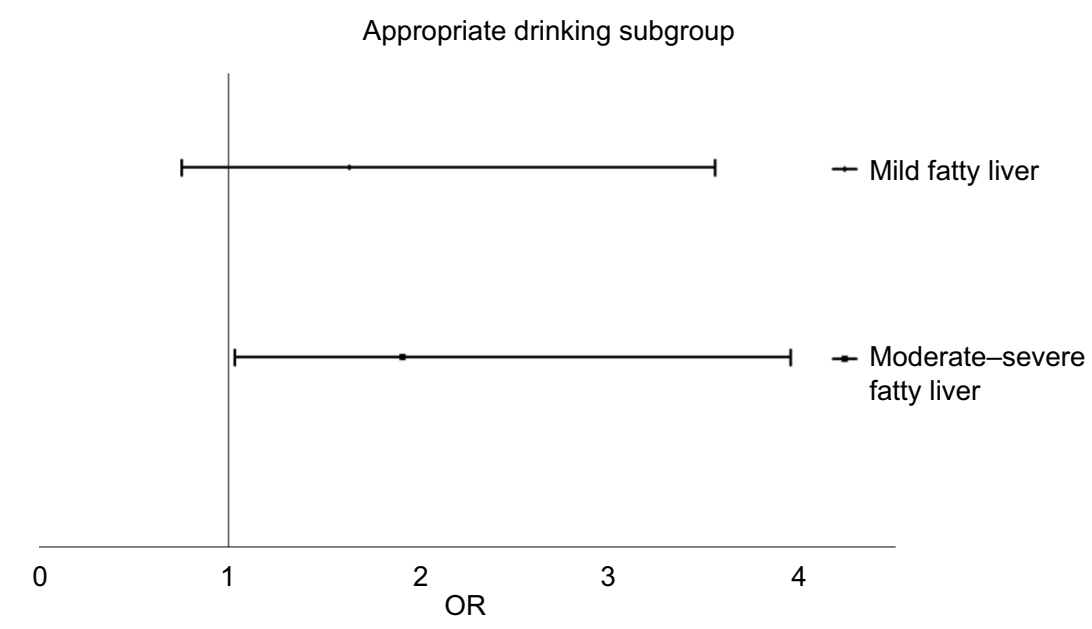

Figure I OR of zonulin concentration in fatty liver disease in the appropriate drinking subgroup.

Notes: In the multivariate multinomial logistic regression analysis adjusted for covariates, the OR of zonulin concentrations in relation to the incidence of mild fatty liver was 1.63 (adjusted $\mathrm{OR}=1.63,95 \% \mathrm{Cl}=0.75-3.55$ ) in the appropriate drinking group and was not statistically significant. However, the OR for moderate-to-severe fatty liver was $1.91(95 \% \mathrm{Cl}=\mathrm{I} .0 \mathrm{I}-3.95)$ in the same group and was statistically significant.

Abbreviations: $\mathrm{OR}$, odds ratio; $\mathrm{Cl}$, confidence interval.

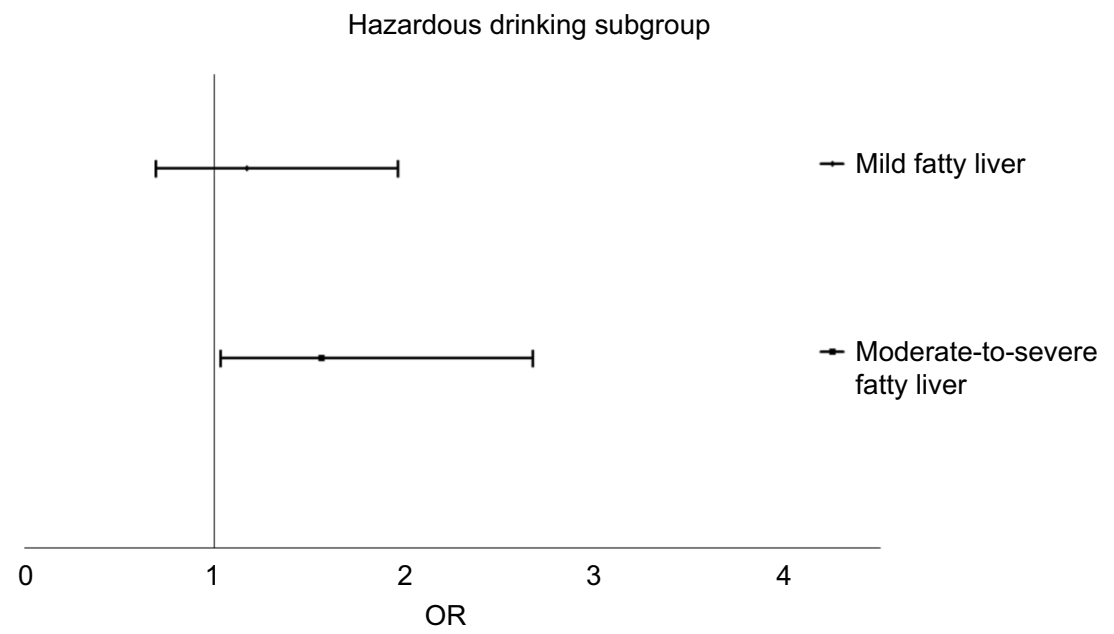

Figure 2 OR of zonulin concentration in fatty liver disease in the hazardous drinking subgroup.

Notes: In the multivariate multinomial logistic regression analysis adjusted for covariates, the OR of zonulin concentrations in relation to the incidence of mild fatty liver was $1.17(95 \% \mathrm{Cl}=0.69-1.96)$ in the hazardous drinking group and was not statistically significant. However, the OR for moderate-to-severe fatty liver was I.56 (95\% $\mathrm{Cl}=\mathrm{I} .02-2.67)$ in the same group and was statistically significant.

Abbreviation: OR, odds ratio.

intestinal inflammation-related obesity leads to increased intestinal permeability. Conversely, increased intestinal permeability leads to increased plasma LPS concentrations and low-grade inflammation, causing insulin resistance and obesity. ${ }^{20}$ Obesity plays an important role in the pathogenesis of fatty liver disease on its own. Insulin resistance resulting from obesity induces chronic hyperinsulinemia, which leads to fat accumulation in the liver. ${ }^{21}$
Peripheral adipose tissue increases because obesity produces excess free fatty acids due to insulin resistance. As a result, fat accumulates in the liver and this in turn aggravates insulin resistance, thus forming a malicious cycle..$^{22}$ Additionally, insulin resistance and elevated levels of saturated free fatty acids resulting from obesity may play an important role in liver cell damage, progression of nonalcoholic fatty liver disease, and fibrosis. ${ }^{23}$ 
In the present study, the plasma zonulin concentrations in obese patients were assessed. We confirmed the known finding that obesity is associated with increased intestinal permeability and plays an important role in the pathogenesis of fatty liver disease. Plasma zonulin concentrations were found to be significantly associated with the severity of fatty liver disease. We found a significant association in participants with both high and low alcohol consumption.

Alcohol consumption is an important risk factor of fatty liver disease. For this reason, most studies that investigated the association between fatty liver disease and intestinal permeability have examined cases of alcoholic liver disease and nonalcoholic liver disease separately. Changes in intestinal permeability are an important mechanism causing severe complications of alcoholic liver diseases..$^{24}$ Persson et al ${ }^{10}$ reported that alcohol consumption induces malfunction of the intestinal walls and increases intestinal permeability. Such et a ${ }^{25}$ reported that patients with advanced cirrhosis have increased intestinal permeability due to increased nitric oxide concentrations caused by portal hypertension, swelling of the intestinal mucosa, and intestinal epithelial ultrastructural changes. One study also demonstrated an association between increased intestinal permeability in patients with alcoholic cirrhosis and spontaneous bacterial infections. ${ }^{26}$ However, no studies have investigated the association between uncomplicated alcoholic liver diseases and intestinal permeability, and further research is needed in this area.

In the present study, zonulin concentrations increased as the severity of fatty liver disease increased in heavy and binge drinkers. This is a significant finding since previous studies involved patients with advanced alcoholic liver diseases, such as cirrhosis. In this study, such an association was found in patients with otherwise uncomplicated fatty liver disease. Considering that alcoholic liver diseases occur only in some consumers of alcohol, ${ }^{24}$ factors aside from alcohol may also affect the pathogenesis of alcoholic liver disease. Metabolic disorders, such as obesity and insulin resistance, are reported to play an important role in the worsening of alcoholic liver disease. ${ }^{27}$ Considering such an association between metabolic disorders and intestinal permeability, examining and managing the mechanisms affecting intestinal permeability in patients with asymptomatic, uncomplicated fatty liver disease may be important as we learned in this study. Moreover, considering the results of the previous study that blockade of the zonulin receptor reduced metabolic disorder associated with increased intestinal permeability, ${ }^{28}$ the results of our study may be the basis for research on the role of zonulin modulators in preventing the development of advanced alcoholic liver disease.

Researchers have paid attention to not only alcoholic liver disease but also the association between nonalcoholic fatty liver disease and intestinal permeability. However, studies on nonalcoholic fatty liver disease and intestinal permeability are sparse and inconclusive. Farrel ${ }^{29}$ revealed that increased gut microbiota leads to increased intestinal permeability and subsequently to increased endotoxin absorption and nonalcoholic fatty liver disease. By contrast, Wigg et $\mathrm{al}^{4}$ reported that although the incidence rate of nonalcoholic small intestine bacterial overgrowth was twice as high, this was not significantly associated with increased intestinal permeability and increased plasma endotoxin concentrations. Pacifico et al ${ }^{30}$ reported that the plasma zonulin concentration, a marker of intestinal permeability, and the severity of nonalcoholic fatty liver disease were significantly associated in their study involving pediatric patients.

In our study, plasma zonulin concentrations increased as the severity of fatty liver disease increased even in social drinkers ( $<14$ standard drinks per week) who are considered to have the nonalcoholic fatty liver disease. We also observed that the risk of severe fatty liver disease of Grade 2 or higher increased as plasma zonulin concentrations increased. A possible mechanism for the association between plasma zonulin concentrations and severity of nonalcoholic fatty liver disease that was seen in these studies is as follows. First, changes in the gut microbiota that occur in obese individuals lead to increased intestinal permeability, which may result in a high plasma zonulin concentration. Second, changes in microbiota may also lead to increased production of inflammatory cytokines. LPS released by intestinal bacteria and the bacteria themselves act as toll-like receptor (TLR) ligands to excessively stimulate TLRs with tolerance. ${ }^{31,32}$ The released inflammatory cytokines cause liver inflammation. In another aspect, Zhu et al ${ }^{132}$ reported that gut microbiota enriched in alcohol-producing bacteria due to obesity constantly produces more alcohol than healthy microbiota and therefore supplies a constant source of reactive oxygen species to the liver. This endogenous alcohol may cause inflammation of the liver. However, since participants in our study were obese patients whose intestinal permeability was already higher than that of normal-weight patients, further research on the importance of intestinal permeability in the pathogenesis of nonalcoholic fatty liver disease is needed.

In the present study, plasma zonulin as a risk factor was significant in moderate-to-severe fatty liver disease but not in mild fatty liver disease. The reason why the OR is $\geq 1$ but 
not statistically significant may be attributed to the relatively small number of subjects with normal livers because all subjects included in this study were obese men. A large-scale study should be conducted in the future.

This study has several limitations. First, in our patients, fatty liver was diagnosed radiologically, not histologically. Since all patients were relatively healthy, biopsy would have been relatively invasive, but ultrasound would have been performed noninvasively at the outpatient clinic. However, previous studies have suggested that ultrasound results are sufficiently correlated with histologic findings. ${ }^{33}$ In view of this fact, the results of this study can be considered meaningful. Second, although patients with previous histories of celiac disease or inflammatory bowel disease were excluded, factors such as gut microbiota and endotoxins were not controlled for. Third, this study was a cross-sectional study and did not investigate the causal relationship between zonulin concentrations and the severity of fatty liver disease. Further research will be required to clarify causal relationships through large-scale prospective studies. Finally, this study was conducted on young men only and cannot provide insight on sex differences in plasma zonulin, and to our knowledge, there has been no prior research in this area. Thus, further studies are needed.

Nevertheless, this study also has several strengths. First, only obese male patients were studied to minimize the influence of obesity, an important factor in intestinal permeability and the pathogenesis of fatty liver disease. Second, this study confirmed the association between zonulin concentrations and the severity of fatty liver disease in Asian men with a relatively high incidence of fatty liver disease.

\section{Conclusion}

Plasma zonulin concentrations in obese men were significantly elevated as the severity of fatty liver disease increased. This elevation was seen in not only hazardous drinkers, but also men who drink less than 14 standard drinks per week.

Based on this result, it may be deduced that changes in intestinal permeability occur in the relatively early stages of liver disease, before progressing to severe liver disease, such as liver cirrhosis. Large-scale prospective research on the causal relationship between changes in intestinal permeability and liver diseases may be necessary. In addition, gaining a sufficient understanding of the pathogenesis of liver diseases associated with increased intestinal permeability and exploring ways to prevent the development and worsening of fatty liver disease may be important.

\section{Acknowledgments}

This work was supported by the grant of Biomedical Research Institute, Kyungpook National University Hospital (2016).

Data collection and analyses were completely performed by the authors; the hospital had no influence on the study design, data acquisition, data analysis, data interpretation, or in writing the manuscript.

\section{Disclosure}

The authors report no conflicts of interest in this work.

\section{References}

1. Madara JL, Moore R, Carlson S. Alteration of intestinal tight junction structure and permeability by cytoskeletal contraction. Am J Physiol. 1987;253(6):C854-C861.

2. DeMeo MT, Mutlu EA, Keshavarzian A, Tobin MC. Intestinal permeation and gastrointestinal disease. J Clin Gastroenterol. 2002;34(4): 385-396.

3. Van Elburg R, Uil J, Mulder C, Heymans H. Intestinal permeability in patients with coeliac disease and relatives of patients with coeliac disease. Gut. 1993;34(3):354-357.

4. Wigg AJ, Roberts-Thomson IC, Dymock RB, McCarthy PJ, Grose RH, Cummins AG. The role of small intestinal bacterial overgrowth, intestinal permeability, endotoxaemia, and tumour necrosis factor alpha in the pathogenesis of non-alcoholic steatohepatitis. Gut. 2001;48(2):206-211.

5. Sapone A, de Magistris L, Pietzak M, et al. Zonulin upregulation is associated with increased gut permeability in subjects with type 1 diabetes and their relatives. Diabetes. 2006;55(5):1443-1449.

6. Kim CH, Younossi ZM. Nonalcoholic fatty liver disease: a manifestation of the metabolic syndrome. Cleve Clin J Med. 2008;75(10):721-728.

7. Bedogni G, Nobili V, Tiribelli C. Epidemiology of fatty liver: an update. World J Gastroenterol. 2014;20(27):9050-9054.

8. Targher G, Day CP, Bonora E. Risk of cardiovascular disease in patients with nonalcoholic fatty liver disease. $N$ Engl J Med. 2010;363(14): 1341-1350.

9. Moreno-Navarrete JM, Sabater M, Ortega F, Ricart W, Fernandez-Real JM. Circulating zonulin, a marker of intestinal permeability, is increased in association with obesity-associated insulin resistance. PLoS One. 2012;7(5):e37160.

10. Persson J, Berg NO, Sjolund K, Stenling R, Magnusson PH. Morphologic changes in the small intestine after chronic alcohol consumption. Scand J Gastroenterol. 1990;25(2):173-184.

11. Everard A, Cani PD. Diabetes, obesity and gut microbiota. Best Pract Res Clin Gastroenterol. 2013;27(1):73-83.

12. Fasano A. Zonulin, regulation of tight junctions, and autoimmune diseases. Ann N Y Acad Sci. 2012;1258:25-33.

13. Wang W, Uzzau S, Goldblum SE, Fasano A. Human zonulin, a potential modulator of intestinal tight junctions. J Cell Sci. 2000;113(pt 24): 4435-4440.

14. El Asmar R, Panigrahi P, Bamford P, et al. Host-dependent activation of the zonulin system is involved in the impairment of the gut barrier function following bacterial colonization. Gastroenterology. 2002;123(5):1607-1615.

15. Drago S, El Asmar R, Di Pierro M, et al. Gliadin, zonulin and gut permeability: effects on celiac and non-celiac intestinal mucosa and intestinal cell lines. Scand J Gastroenterol. 2006;41(4):408-419.

16. Levey AS, Bosch JP, Lewis JB, Greene T, Rogers N, Roth D. A more accurate method to estimate glomerular filtration rate from serum creatinine: a new prediction equation. Modification of Diet in Renal Disease Study Group. Ann Intern Med. 1999;130(6):461-470.

17. Saadeh S, Younossi ZM, Remer EM, et al. The utility of radiological imaging in nonalcoholic fatty liver disease. Gastroenterology. 2002;123(3):745-750. 
18. Lam YY, Ha CW, Campbell CR, et al. Increased gut permeability and microbiota change associate with mesenteric fat inflammation and metabolic dysfunction in diet-induced obese mice. PLoS One. 2012;7(3):e34233.

19. Muccioli GG, Naslain D, Backhed F, et al. The endocannabinoid system links gut microbiota to adipogenesis. Mol Syst Biol. 2010;6:392.

20. Wellen KE, Hotamisligil GS. Inflammation, stress, and diabetes. J Clin Invest. 2005;115(5):1111-1119.

21. Cusi K. Role of insulin resistance and lipotoxicity in non-alcoholic steatohepatitis. Clin Liver Dis. 2009;13(4):545-563.

22. Arner $P$. The adipocyte in insulin resistance: key molecules and the impact of the thiazolidinediones. Trends Endocrinol Metab. 2003;14(3):137-145.

23. Neuschwander-Tetri BA. Hepatic lipotoxicity and the pathogenesis of nonalcoholic steatohepatitis: the central role of nontriglyceride fatty acid metabolites. Hepatology. 2010;52(2):774-788.

24. Bellentani S, Saccoccio G, Costa G, et al. Drinking habits as cofactors of risk for alcohol induced liver damage. Gut. 1997;41(6):845-850.

25. Such J, Guardiola JV, de Juan J, et al. Ultrastructural characteristics of distal duodenum mucosa in patients with cirrhosis. Eur J Gastroenterol Hepatol. 2002;14(4):371-376.

26. Scarpellini E, Valenza V, Gabrielli M, et al. Intestinal permeability in cirrhotic patients with and without spontaneous bacterial peritonitis: is the ring closed? Am J Gastroenterol. 2010;105(2):323-327.
27. Mezey E. Dietary fat and alcoholic liver disease. Hepatology. 1998;28(4):901-905.

28. Watts T, Berti I, Sapone A, et al. Role of the intestinal tight junction modulator zonulin in the pathogenesis of type I diabetes in BB diabeticprone rats. Proc Natl Acad Sci U S A . 2005;102(8):2916-2921.

29. Farrell G. Is bacterial ash the flash that ignites NASH? Gut. 2001;48(2):148-149.

30. Pacifico L, Bonci E, Marandola L, Romaggioli S, Bascetta S, Chiesa C. Increased circulating zonulin in children with biopsy-proven nonalcoholic fatty liver disease. World J Gastroenterol. 2014;20(45): 17107-17114

31. Ruiz AG, Casafont F, Crespo J, et al. Lipopolysaccharide-binding protein plasma levels and liver TNF-alpha gene expression in obese patients: evidence for the potential role of endotoxin in the pathogenesis of nonalcoholic steatohepatitis. Obes Surg. 2007;17(10):1374-1380.

32. Zhu L, Baker SS, Gill C, et al. Characterization of gut microbiomes in nonalcoholic steatohepatitis (NASH) patients: a connection between endogenous alcohol and NASH. Hepatology. 2013;57(2):601-609.

33. Yajima Y, Ohta K, Narui T, Abe R, Suzuki H, Ohtsuki M. Ultrasonographical diagnosis of fatty liver: significance of the liver-kidney contrast. Tohoku J Exp Med. 1983;139(1):43-50.
Diabetes, Metabolic Syndrome and Obesity: Targets and Therapy is an international, peer-reviewed open-access journal committed to the rapid publication of the latest laboratory and clinical findings in the fields of diabetes, metabolic syndrome and obesity research. Original research, review, case reports, hypothesis formation, expert opinion and commentaries are all considered for publication. The manuscript management system is completely online and includes a very quick and fair peer-review system, which is all easy to use. Visit http://www.dovepress.com/testimonials.php to read real quotes from published authors. 